\title{
Study on Evaluation and Training of Communication Ability between Doctors and Patients among Clinical Medical Postgraduates in Higher Medical Education
}

Mengqi Tang ${ }^{1,2,3, a}$, Zhiqiang Feng ${ }^{1,2,3, b}$, Junwei Song ${ }^{1,2,3, c}$, Changhai Tang $^{1,2,3, d}$, Lili Zhu ${ }^{1,2,3, \mathrm{e}}$, Zhongming Chen ${ }^{1,2,3, \mathrm{f}}$, Wei Wang ${ }^{1,2,3, \mathrm{~g}}$, Wenqiang Yin ${ }^{2,3,4, \mathrm{~h}^{*}}$

${ }^{1}$ School of Public Health and Management, Weifang Medical University, Weifang 261053, China;

${ }^{2}$ Collaborative Innovation Center of Social Risks Governance in Health, Shanghai 200032, China.

3“Health Shandong" Severe Social Risk Prevention and Management Synergy Innovation Center, Weifang 261053, China;

${ }^{4}$ Department of Public Course, Weifang Medical University, Weifang 261053, China.

atmqpsy@163.com, bfengzhiqiang7@163.com, 'sjwsongjunwei@163.com, ${ }^{d} 15056041071 @ 163 . c o$ m, ${ }^{\text {} z l l w f y x y @ f o x m a i l . c o m, ~}{ }^{\mathrm{f}} \mathrm{czm} 3306196 @ 163 . c o m,{ }^{\mathrm{g}}$ ann2006@163.com, ${ }^{\mathrm{h}}$ yinwq1969@126.com.

${ }^{*}$ Corresponding author

Keywords: Higher medical education, Clinical medical graduate students, Doctor-patient communication ability, Comprehensive evaluation, Training.

Abstract. Objective Through the comprehensive evaluation of doctor-patient communication ability of the clinical medical postgraduates in all grades of medical colleges and universities, it provides a basis for the cultivation and promotion of better communication ability between doctors and patients in clinical medicine postgraduates. Methods By using RSR method and cluster analysis method synthetically evaluates and analyzes the communication ability of medical postgraduates in three grades of three medical colleges and universities in Shandong province. Results The average score of communication ability between doctors and patients in clinical medicine postgraduate is 3.875. In the RSR analysis and cluster analysis, the proportion of "poorer" and "poorest" clinical medicine postgraduates is $17.1 \%$ and $8.3 \%$ respectively. Conclusion Part of the clinical medical postgraduates doctor-patient communication ability are still need to be improved. The colleges should focus on the links of communication feedback and environmental perception, to strengthen the training of the doctor-patient communication ability of clinical medical postgraduates.

\section{Introduction}

Good communication skills between doctors and patients is an important guarantee for communication between medical staff and patients [1]. In recent years, the contradiction between doctors and patients in China is outstanding, and medical disputes are continually occurring. Various violent injuries happened frequently [2]. Studies have shown that the lack of communication between doctors and patients is one of the main contradictions between them [3, 4]. As the future medical practitioner, the training of doctor-patient communication ability among clinical medicine graduate students will play an important role in the higher medical education at present.

\section{Materials and Methods}

\subsection{Sources of Information}

In this study, a multi-stage stratified random sampling method is used to divide the medical colleges into two layers according to the school situation. One or two schools are selected into each layer. Each medical school is divided into three layers according to the grade, respectively, ranging 
from the first grade to the third grade. Each grade is randomly selected part of the clinical medical graduate students to conduct a questionnaire survey. We shall use the task group to design the questionnaire survey. A total of 990 questionnaires were sent out, 974 were collected and the recovery rate was $98.38 \%$. The effective questionnaires were 929 and the effective rate was $95.38 \%$.

\subsection{Research Tools}

The postgraduates' communication ability questionnaire includes demographic variables, doctor-patient communication ability assessment questionnaire. Among them, the ability of communication between doctors and patients is analyzed by 68 questions, and the results are analyzed by using Likert's five-level method, namely 1 point (very nonconformity), 2 points (nonconformity), 3 (general), 4 points (consistent), 5 points (very consistent), scores are 1,2,3,4,5[5] The reliability test shows that the Cronbach's $\alpha$ coefficient is 0.982, and the internal consistency coefficient is $0.833 \sim 0.968$, which indicates that the reliability of the questionnaire has good reliability. The structure validity analysis shows that, KMO is 0.982 through exploratory factor analysis (EFA), Bartlett's test of sphericity is $\chi^{2}=47688.261, P<0.001$, which showed that it is suitable for factor analysis. According to the results of the analysis, we extracts seven common factors from 68 entries, which are summed up as communication plan and preparation dimension ( $\mathrm{f}_{1}$, with 6 items), communication initiation dimension ( $\mathrm{f}_{2}$, with 8 items), information transmission dimension( $\mathrm{f}_{3}$, with 26 items), communication feedback dimension ( $\mathrm{f}_{4}$, with 8 entries), environmental perception dimension ( $f_{5}$, with 11 entries), communication noise dimension ( $f_{6}$, with 5 entries) and communication end dimension ( $f_{7}$, with 4 entries). The explanatory power of the 7 common factors to the total variation is $63.536 \%$, which indicates that it has good construct validity.

\subsection{Research Methods}

In this study, RSR analysis method and clustering analysis are used to evaluate the communication ability of postgraduates in clinical medicine. The communication planning and preparation, communication initiation, information transmission, communication feedback, environment perception, communication noise and communication end indicators are used to evaluate the communication ability of postgraduates in clinical medicine. The seven indicators are high excellent indicators. The greater the index is, the better communication ability of clinical post-graduate students will be.

The rank sum ratio method is a multi-index comprehensive evaluation method proposed by Prof. Tian Fengtiao in 1988. It combines the information of multiple evaluation indicators and shows the comprehensive level of multiple evaluation indexes [6]. Clustering analysis is a statistical method of classification of random phenomena, by directly comparing the properties of samples or indicators, the similar nature will be classified as a class, and different properties are classified in different categories, making all types of within the variation is small, large variation between classes [7].

\section{Results}

\subsection{The doctor-patient communication ability of clinical medicine graduate students}

As shown in Table 1, seven dimensions of communication planning and preparation, communication initiation, information transmission, communication feedback, environment perception, communication noise, and communication end are analyzed for three grades of clinical medical postgraduates. Then the communication ability score is obtained. Communication plan and preparation dimension average score is 3.976, communication initiation dimension average score is 3.858, information transmission dimension average score is 4.028, communication feedback dimension average score is 3.762, environmental perception dimension average score is 3.729, communication noise dimension average score is 3.831, the communication end dimension average score is 3.865 , the overall average score is 3.875 . 


\subsection{Comprehensive evaluation of communication ability of postgraduates in clinical medicine} 3.2.1 To evaluate the doctor-patient communication ability of clinical medical graduate students by RSR method

Firstly, based on the RSR method, with the survey of 3 medical colleges and universities in the three grades of clinical medical graduate student doctor-patient communication skills of communication planning and preparation, communication initiation, information transmission, communication feedback, environment perception, communication noise, and communication end, we list "each grade of clinical medical students ' doctor-patient communication ability data table". According to the seven selected indexes, the grade scores of the three grades are ranked. As the indexes are all high-grade indexes, the rank should be in order from small to large [8], as shown in Table 1.

Secondly, according to the formula $R S R_{i}=\sum_{j=1}^{m} R_{i j} / n k(k$ is the evaluation index, $n$ is the evaluation object, $R_{i j}$ is the $i$-th row, $j$ element of rank), we get the grade of the rank sum ratio calculation formula of clinical medical graduate communication ability: $R S R$ Doctor-patient communication capacity $=\left(R_{1}+R_{2}+R_{3}+R_{4}+R_{5}+R_{6}+R_{7}\right) / n k$. Using the formula of rank sum ratio, the RSR value of communication ability between doctors and patients in clinical medicine graduate students is calculated. And then reorder the RSR of the doctor-patient communication ability of the postgraduates of clinical medicine in each grade (Table 1).

Table 1 The datasheet of doctor-patient communication ability of clinical medical postgraduates in different grades

\begin{tabular}{cccccccccc}
\hline Grade & $\begin{array}{c}\text { Communication } \\
\text { plan }\end{array}$ & $\begin{array}{c}\text { Communication } \\
\text { initiation }\end{array}$ & $\begin{array}{c}\text { Information } \\
\text { transmission }\end{array}$ & $\begin{array}{c}\text { Communication } \\
\text { feedback }\end{array}$ & $\begin{array}{c}\text { Environmental } \\
\text { perception }\end{array}$ & $\begin{array}{c}\text { Communication } \\
\text { noise }\end{array}$ & $\begin{array}{c}\text { Communication } \\
\text { end }\end{array}$ & RSR & Rank \\
\hline G1 & $4.003(3)$ & $3.850(1)$ & $\begin{array}{c}4.032 \\
(2)\end{array}$ & $3.748(1)$ & $3.733(2)$ & $3.804(1)$ & $3.853(1)$ & 0.6190 & 2 \\
G2 & $3.927(1)$ & $3.847(2)$ & $\begin{array}{c}4.020 \\
(1)\end{array}$ & $3.767(2)$ & $3.712(1)$ & $3.881(3)$ & $3.861(2)$ & 0.5238 & 3 \\
G3 & $3.978(2)$ & $3.884(3)$ & $\begin{array}{c}4.026 \\
(3)\end{array}$ & $3.784(3)$ & $3.738(3)$ & $3.823(2)$ & $3.893(3)$ & 0.8571 & 1 \\
$\overline{\mathrm{X}}$ & 3.976 & 3.858 & 4.028 & 3.762 & 3.729 & 3.831 & 3.865 & - & - \\
\hline
\end{tabular}

\subsubsection{Clustering analysis}

Based on the average method and the RSR analysis index, 929 clinical medical graduate students' communication ability are clustered and divided into five groups. In the clustering results, the proportion of "general" communication students is the highest, accounting for $47.5 \%$; followed by "poorer" communication students, accounting for $17.1 \%$; the communication ability of "best”, "better" and "poorest” accounting for 15.6\%, 11.5\% and 8.3\% respectively. Thus, the ability of clinical medicine graduate students to communicate more concentrated in the "general" and "poor" category, as shown in Table 2.

Table 2 Clustering analysis of the doctor-patient communication ability of clinical medicine graduate students

\begin{tabular}{cccccc}
\hline \multirow{2}{*}{ Project } & \multicolumn{5}{c}{ Classification } \\
\cline { 2 - 6 } & Best & Better & General & Poorer & Poorest \\
\hline$R S R$ & $\geq 0.768$ & $0.643-0.759$ & $0.327-0.637$ & $0.170-0.324$ & $<0.004$ \\
Constituent ratio (\%) & 15.6 & 11.5 & 47.5 & 17.1 & 8.3 \\
Cumulative percentage (\%) & 15.6 & 27.1 & 74.6 & 91.7 & 100 \\
\hline
\end{tabular}

\section{Discussion}

As an important part of higher education, the educational content and quality of higher medical 
education will affect both the higher education and health system development [9]. In the higher medical education, as the future medical workers, the ability of communication between doctors and patients of clinical medicine graduate students occupies an important position. The communication ability of doctors and patients at present will affect the future development of doctor-patient relationship and the construction of harmonious doctor-patient relationship. Therefore, it is necessary to analyze the communication ability of doctors and patients in clinical medicine graduate students from the communication process of doctors and patients, and to increase the communication ability of postgraduates and improve the communication ability of postgraduates in higher medical education.

\subsection{Analysis of the doctor-patient communication ability in graduate students of clinical medicine}

This study starts from the seven links of doctor-patient communication process and analyzes the communication ability of doctor-patient communication of clinical medicine graduate students. By analyzing the score of communication ability between doctors and patients in clinical medicine graduate students, the average score of doctor-patient communication ability is 3.875 , which is moderate. The communication ability of doctor-patient among the three grades of clinical medicine postgraduates was analyzed by RSR and cluster analysis. Most of their doctor-patient communication ability is in "general" level. But there are still $25.4 \%$ of postgraduates communication ability is poor. It indicates that there is still room for improvement in communication ability of postgraduates in clinical medicine. Therefore, we should strengthen the training of doctor-patient communication ability for clinical medicine postgraduates in higher medical education.

\subsection{The training of doctor-patient communication ability of clinical medicine graduates in higher medical education}

The analysis of the results shows that the postgraduates' communication feedback and environmental perception dimension scores are low. So we should focus on cultivating their communication ability of doctors and patients from these two aspects. In order to improve the ability of communication between doctors and patients, the training mode of communication between doctors and patients in clinical medicine should be perfected together in medical colleges and universities and teaching hospitals. Communication feedback is mainly in the process of communication, clinical medicine graduate students can accurately grasp the needs, psychological status and role of patient, and accurately judge them through the patient's facial expressions and body language. At the same time they can timely adjust their communication way according to the patient's response. And even when an emergency occurs, they can properly communicate with patients. Therefore, in the stage of clinical medicine graduate students' theoretical study, medical colleges and universities should standardize the establishment of doctor-patient communication course and strengthen the impartation of communication skills between doctors and patients. In the teaching hospital, clinical teaching teachers should pay attention to the clinical practice training of postgraduates, and increase the communication opportunity with patients and their families to enhance the "empathy" training, accurately grasp of the need of patients, improve patient confidence to the postgraduates, in order to better communicate and improve the treatment effect. In environmental perception, clinical medicine graduate students should fully use the variety of information which is collected by communicating with patients, and communicate with different patients in different ways by the help of different region, occupational, cultural background, age and other factors of patients. They should also learn to use charts, mannequin and other tools to communicate with patients, to help patients better understand their own condition, and to strengthen their understanding of their disease.

\section{Acknowledgements}

This paper is sponsored by the Innovation Projects of Graduate Student Education in Shandong Province, China (GN: SDYY14120); the $12^{\text {th }}$ Five-Year Plan Project of Education Science in Shandong Province, China (GN: 2011GG293). Corresponding author of this paper is professor 
Wenqiang Yin.

\section{References}

[1] Youjia $\mathrm{Xu}$, Zhenfang Zhang. The ability of physician-patient communication is an important guarantee for a haimonious physician-patient relationship[J]. China Health Law, 2007,01:32-34.

[2] Rui Han, Weili Shi, Yanlin Li, et al. The important value of the communication skills between doctors and patients in clinical medical students[J]. China Medical Herald, 2015,35:140-143.

[3] Shi Yin. Tension and its causes of relationship between the medical and the patient[J]. Health Economics Research, 2003,02:14-16.

[4] Elizabeth Weise. Doctor - patient rapport lacking and both agree communication canbe matter of life or deat [N]. USA Today, 2010-06-12.

[5] Wei Wang, Wenqiang Yin. Development of Interpersonal Communication Ability Scale for Medical Students[J]. Chinese General Practice, 2015,18(22): 2709-2712.

[6] Haiyi Jia, Wenqiang Yin, Zhongming Chen, et al. To evaluate the prevention and health care services of township hospitals in Shandong Province with RSR and TOPSIS analysis methods[J].Chinese Journal of Health Statistics,2015,03:427-429.

[7] Zhenqiu Sun, Lesan Wang. Comprehensive evaluation method and its application in medicine[M]. Beijing: Chemical Industry Press, 2006: 75.

[8] Zhongming Chen. The study on situation of outpatient medication in township hospitals of Shandong province under the background of essential medicine system[D]. Weifang: Weifang Medical University, 2014.

[9] Yan Li, Liyang Wen. Studies on structure of higher medical education in China[J]. China Higher Medical Education, 2012,01: 1-4, 20. 